\title{
PITUITARY CORTICOTROPHIN (ACTH) PRODUCTION IN RHEUMATOID ARTHRITIS TESTED INDIRECTLY WITH METOPIRON (SU 4885)
}

\author{
BY \\ J. L. KALLIOMÄKI, N. T. KÄRKI, H. A. SAARIMAA, AND E. TALA \\ From the Departments of Medicine and Pharmacology, University of Turku, Finland
}

Metopiron [2-methyl-1,2-bis-3-pyridyl)-1-propanone; $\mathrm{Su} 4885]$ inhibits $11-\beta$-hydroxylation in the adrenal cortex, and this inhibition is reflected as an increased production of ACTH in the anterior pituitary. It was found by Liddle, Estep, Kendall, Williams, and Townes (1959) that the pituitary production of ACTH in man can be measured with aid of Metopiron and numerous clinical studies have since been carried out which have shown that the Metopiron test can be employed as a reliable indirect method of evaluating the pituitary production of ACTH (e.g. Andersson, 1961; Faber, 1961; Gold, Kent, and Forsham, 1961).

In this paper we report results that obtained when the Metopiron test was applied to patients with rheumatoid arthritis.

\section{Material and Methods}

Our rheumatoid group comprised ten patients ( 3 male, 7 female; average age 53.7 yrs) who had not received steroid treatment for one year before the experiment.

The control group also comprised ten patients ( 3 male, 7 female; average age $46.0 \mathrm{yrs}$ ); the diagnoses of these patients are shown in Table II.

The patients in both groups were hospitalized during the whole experimental period of 6 days. The first, second, fifth, and sixth days were control days when Metopiron was not administered. On the third and fourth days the patients were all given $500 \mathrm{mg}$. Metopiron orally every 6 hours.

The 24-hr urine was collected and analysed for total 17-hydroxycorticosteroids (17-OHCS) by a modification of the method of Jenkins, Forsham, Laidlaw, Reddy, and Thorn (1955).

\section{Results}

The results of the Metopiron tests for the rheumatoid and control groups are summarized in Tables I and II (opposite).

The differences between the mean values for the two groups are not statistically significant.
A tendency for a delayed reaction in the Metopiron test was evident in the rheumatoid group. Thi maximal average excretion of total 17-OHCS wast recorded in the rheumatoid group on the fifth days (the day following the Metopiron administration) $\vec{\overrightarrow{ }}$ whereas it was recorded on the fourth day (the second day of Metopiron administration) in the control group.

\section{Discussion}

Our results seem to indicate that in rheumatid patients the capacity of the pituitary to prodi $\mathrm{ACTH}$, as determined indirectly by the exogen inhibition of $11-\beta$-hydroxylation in the adrenas cortex, does not differ from the capacity in patient with various "non-collagenous" diseases. A ten dency was noted in the rheumatoid patients for the response of the pituitary to be delayed, but it was not possible to determine on the basis of our results whether this finding was accidental or not. In earlier study (Kalliomäki and Rauramo, 1960): it was found that urinary excretion of gonado $\frac{\stackrel{2}{2}}{2}$ trophins is normal in female rheumatoid patientsSigns suggesting the thyrohypophyseal syndrome and an increased extracellular phase in rheumatoid arthritis (Kalliomäki and Holopainen, 1956; Kallio mäki, Kirpilä, Koskinen, and Laine, 1958) mayo reflect disturbances in the hypothalamo-pituitary system. It is possible that the delayed response of the anterior pituitary gland in the Metopiron tes is also a manifestation of the same kind.

Metopiron has weak diuretic properties (iN inhibits aldosterone production). Tables I and IN show that the average total output of urine of the rheumatoid patients on the third, fourth, and fiftif days was about half a litre larger than the average output in the control group. This may be takerop as an indication of the above-mentioned pre $=$ disposition to an increase in the extracellular fluide 
TABLE I

RESULTS OF METOPIRON TEST IN THE RHEUMATOID GROUP

\begin{tabular}{|c|c|c|c|c|c|c|c|c|c|c|c|}
\hline \multirow{4}{*}{$\begin{array}{l}\text { Case } \\
\text { No. }\end{array}$} & \multirow{4}{*}{ Sex } & \multirow{4}{*}{$\begin{array}{l}\text { Age } \\
\text { (yrs) }\end{array}$} & \multirow{4}{*}{$\begin{array}{c}\text { History } \\
\text { of } \\
\text { Rheumatoid } \\
\text { Arthritis } \\
\text { (yrs) }\end{array}$} & \multirow{4}{*}{$\begin{array}{c}\text { Stage of } \\
\text { Rheumatoid } \\
\text { Arthritis }\end{array}$} & \multirow{4}{*}{$\begin{array}{c}\text { Erythrocyte } \\
\text { Sedimen- } \\
\text { mentation } \\
\text { Rate } \\
\text { (mm./hr) }\end{array}$} & \multicolumn{6}{|c|}{ Urinary Excretion of Total 17-OHCS (mg./24 hrs) } \\
\hline & & & & & & \multicolumn{6}{|c|}{ Days } \\
\hline & & & & & & \multicolumn{2}{|c|}{ Control } & \multicolumn{2}{|c|}{ Metopiron } & \multicolumn{2}{|c|}{ Control } \\
\hline & & & & & & 1 & 2 & 3 & 4 & 5 & 6 \\
\hline $\begin{array}{r}1 \\
2 \\
3 \\
4 \\
5 \\
6 \\
7 \\
8 \\
9 \\
10\end{array}$ & $\begin{array}{l}\mathbf{M} \\
\mathbf{M} \\
\mathbf{M} \\
\mathbf{F} \\
\mathbf{F} \\
\mathbf{F} \\
\mathbf{F} \\
\mathbf{F} \\
\mathbf{F} \\
\mathbf{F}\end{array}$ & $\begin{array}{l}44 \\
33 \\
52 \\
38 \\
63 \\
69 \\
55 \\
67 \\
19 \\
50\end{array}$ & $\begin{array}{r}30 \\
5 \\
27 \\
1 / 4 \\
1 / 3 \\
8 \\
8 \\
30 \\
10 \\
17\end{array}$ & $\begin{array}{l}\text { III } \\
\text { III } \\
\text { III } \\
\text { II } \\
\text { II } \\
\text { III } \\
\text { IV } \\
\text { IV } \\
\text { IV } \\
\text { III }\end{array}$ & $\begin{array}{r}100 \\
60 \\
84 \\
117 \\
74 \\
43 \\
31 \\
50 \\
20 \\
54\end{array}$ & $\begin{array}{r}19.2 \\
1.8 \\
2.0 \\
5.9 \\
8.4 \\
2.5 \\
11.0 \\
4.8 \\
4.5 \\
3.1\end{array}$ & $\begin{array}{r}12.6 \\
3.4 \\
6.2 \\
2 \cdot 1 \\
5.6 \\
2.9 \\
4 \cdot 6 \\
1 \cdot 3 \\
2 \cdot 3 \\
2 \cdot 3\end{array}$ & $\begin{array}{r}12 \cdot 8 \\
9.1 \\
5.4 \\
8 \cdot 4 \\
9 \cdot 5 \\
9 \cdot 2 \\
11 \cdot 2 \\
8.8 \\
10 \cdot 3 \\
4 \cdot 3\end{array}$ & $\begin{array}{r}6 \cdot 8 \\
5 \cdot 8 \\
6 \cdot 5 \\
9 \cdot 2 \\
12.0 \\
6.4 \\
18.6 \\
5.8 \\
13.9 \\
4 \cdot 1\end{array}$ & $\begin{array}{r}16.0 \\
2.6 \\
2.4 \\
13.9 \\
7.6 \\
8.4 \\
12.9 \\
11.8 \\
13.0 \\
4.9\end{array}$ & $\begin{array}{l}9 \cdot 8 \\
3 \cdot 4 \\
3 \cdot 7 \\
2 \cdot 0 \\
3 \cdot 7 \\
2 \cdot 4 \\
8 \cdot 0 \\
6 \cdot 9 \\
1 \cdot 7 \\
7 \cdot 0\end{array}$ \\
\hline \multicolumn{5}{|c|}{ Mean Total 17-OHCS Excreted (mg./24 hrs) .. } & $\cdots$ & $6 \cdot 3$ & $4 \cdot 3$ & $8 \cdot 9$ & $8 \cdot 9$ & $9 \cdot 4$ & $4 \cdot 9$ \\
\hline \multicolumn{4}{|c|}{ Average Volume 24-hrs Urine (ml.) } & . & $\cdots$ & 1,080 & 990 & 1,415 & 1,105 & 1,210 & 925 \\
\hline
\end{tabular}

TABLB II

RESULTS OF METOPIRON TEST IN THE CONTROL GROUP

\begin{tabular}{|c|c|c|c|c|c|c|c|c|c|c|c|c|}
\hline \multirow{4}{*}{$\begin{array}{l}\text { Case } \\
\text { No. }\end{array}$} & \multirow{4}{*}{ Sex } & \multirow{4}{*}{$\begin{array}{l}\text { Age } \\
\text { (yrs) }\end{array}$} & \multirow{4}{*}{\multicolumn{2}{|c|}{ Diagnosis }} & & & \multicolumn{6}{|c|}{ Urinary Excretion of Total 17-OHCS (mg./24 hrs) } \\
\hline & & & & & & & \multicolumn{6}{|c|}{ Days } \\
\hline & & & & & & & \multicolumn{2}{|c|}{ Contro! } & \multicolumn{2}{|c|}{ Metopiron } & \multicolumn{2}{|c|}{ Control } \\
\hline & & & & & & & 1 & 2 & 3 & 4 & 5 & 6 \\
\hline $\begin{array}{r}1 \\
2 \\
3 \\
4 \\
5 \\
6 \\
7 \\
8 \\
9 \\
10\end{array}$ & $\begin{array}{l}\mathbf{M} \\
\mathbf{M} \\
\mathbf{M} \\
\mathbf{F} \\
\mathbf{F} \\
\mathbf{F} \\
\mathbf{F} \\
\mathbf{F} \\
\mathbf{F} \\
\mathbf{F}\end{array}$ & $\begin{array}{l}64 \\
56 \\
43 \\
54 \\
62 \\
52 \\
47 \\
34 \\
66 \\
59\end{array}$ & $\begin{array}{l}\text { Cardiac infarction } \\
\text { Cardiac infarction } \\
\text { Cardiac infarction } \\
\text { "Little stroke" .. } \\
\text { Peptic ulcer } \\
\text { Angina pectoris } \\
\text { Acute infection .. } \\
\text { Hypochromic anaemia } \\
\text { Pleural carcinosis } \\
\text { Hodgkin's disease }\end{array}$ & $\begin{array}{l}\cdots \\
\cdots \\
\cdots \\
\cdots \\
\cdots \\
\cdots \\
\cdots\end{array}$ & $\begin{array}{l}\cdots \\
\cdots \\
\cdots \\
\cdots \\
\cdots \\
\cdots \\
\cdots\end{array}$ & \begin{tabular}{l|}
$\cdots$ \\
$\cdots$ \\
$\cdots$ \\
$\cdots$ \\
$\cdots$ \\
$\cdots$ \\
$\cdots$ \\
$\cdots$
\end{tabular} & $\begin{array}{r}4 \cdot 3 \\
3 \cdot 2 \\
4 \cdot 0 \\
13 \cdot 5 \\
7 \cdot 2 \\
3 \cdot 4 \\
8 \cdot 2 \\
6 \cdot 0 \\
12 \cdot 0 \\
4 \cdot 1\end{array}$ & $\begin{array}{r}4 \cdot 0 \\
1 \cdot 8 \\
6 \cdot 0 \\
5 \cdot 9 \\
7 \cdot 2 \\
1 \cdot 4 \\
5 \cdot 8 \\
4 \cdot 3 \\
13 \cdot 9 \\
5 \cdot 0\end{array}$ & $\begin{aligned} 12 \cdot 2 \\
2 \cdot 1 \\
5 \cdot 2 \\
13 \cdot 2 \\
8 \cdot 1 \\
4 \cdot 4 \\
8 \cdot 6 \\
4 \cdot 2 \\
17 \cdot 3 \\
9 \cdot 2\end{aligned}$ & $\begin{aligned} 10 \cdot 5 \\
1 \cdot 4 \\
8 \cdot 4 \\
19 \cdot 7 \\
6 \cdot 9 \\
7 \cdot 2 \\
7 \cdot 2 \\
9 \cdot 7 \\
14 \cdot 0 \\
13 \cdot 6\end{aligned}$ & $\begin{array}{r}5 \cdot 2 \\
0 \cdot 8 \\
8 \cdot 4 \\
13 \cdot 5 \\
6 \cdot 7 \\
3 \cdot 2 \\
5 \cdot 6 \\
3 \cdot 8 \\
20 \cdot 0 \\
13 \cdot 8\end{array}$ & $\begin{array}{c}\text { lacking } \\
\text { lacking } \\
2 \cdot 1 \\
4 \cdot 5 \\
4 \cdot 6 \\
1 \cdot 4 \\
5 \cdot 4 \\
\text { lacking } \\
10 \cdot 0 \\
10 \cdot 4\end{array}$ \\
\hline \multicolumn{4}{|c|}{ Mean Total 17-OHCS Excreted (mg./24 hrs) } & $\cdots$ & $\cdots$ & $\cdots$ & $6 \cdot 6$ & $5 \cdot 5$ & $8 \cdot 5$ & $9 \cdot 9$ & $8 \cdot 1$ & $5 \cdot 5$ \\
\hline \multicolumn{4}{|c|}{ Average Volume 24-hrs Urine (ml.) } & $\cdots$ & .. & $\ldots$ & 1,185 & 840 & 1,238 & 1,095 & $1,000]$ & 920 \\
\hline
\end{tabular}

phase and to increased oedema in rheumatoid arthritis.

\section{Summary}

The variation of the production of ACTH by the pituitary gland when 11- $\beta$-hydroxylation in the adrenal cortex is inhibited by Metopiron ("Su4885") has been followed by determining the mean daily excretion of total 17-hydroxycorticosteroids in ten patients with rheumatoid arthritis and in ten control patients with various internal diseases.

The response of the pituitary gland did not differ essentially in the rheumatoid and control groups, although a tendency for the response to be delayed was noted in the patients with rheumatoid arthritis.

\section{REFERENCES}

Andersson, E. (1961). Ugeskr. Laeg., 123, 223.

Faber, V. (1961). Ibid., 123, 227.

Gold, E. M., Kent, J. R., and Forsham, P. H. (1961). Ann. intern. Med., 54, 175.

Jenkins, D., Forsham, P. H., Laidlaw, J. C., Reddy, W. J., and Thorn, G. W. (1955). Amer. J. Med., $18,3$.

Kalliomäki, J. L., and Holopainen, T. E. (1956). Acta rheum. scand., 2, 213.

Kirpilä, J., Koskinen, H.-M., and Laine, V. A. I. (1958). Ibid., 4, 79.

and Rauramo, L. (1960). Acta endocrinol. (Kbh.), $34,45$.

Liddle, G. W., Estep, H. L., Kendall, J. W., Williams, W. C., and Townes, A. W. (1959). J. clin. Endocrinol., 19, 875. 
Production de corticotrophine (ACTH) dans l'arthrite rhumatismale sous l'influence indirecte de Metopiron (Su 4885)

RÉSUMÉ

On étudia les variations dans la formation d'ACTH par l'hypophyse après l'inhibition de l'hydroxylation en position 11-B dans l'écorce surrénale par Metopiron (Su 4885), en déterminant l'élimination moyenne totale par jour des 17-hydroxycorticostéroïdes chez dix malades atteints d'arthrite rhumatismale et dix témoins souffrant de diverses autres maladies.

La réponse hypophysaire ne révéla pas de différence essentielle entre le groupe rhumatismal et le groupe témoin, mas chez les malades atteints d'arthrite rhumatismale on nota une tendence à répondre tardivement.
Producción de corticotrofina hipofisaria (ACTH) suscitada indirectamente en artritis reumatoide con Metopiron (Su 4885)

\section{SUMARIO}

Se estudiaron las variaciones en la producción de ACTH por la hipófisis, despuès de inhibir la 11-B-e hidroxilación en la corteza suprarrenal con Metopiron (Su 4665), determinando la eliminación media diaria de 17-hidroxocorticosteroides en diez enfermos cond artritis reumatoide $y$ en diez testigos padeciendo de diversas otras enfermedades.

La respuesta hipofisaria no presentó ninguna diferencia esencial entre el grupo con artritis reumatoide y el de control, aunque en los enfermos con artritis reumatoide $\overrightarrow{-}$ se notó tendencia a respuestas retardadas. 